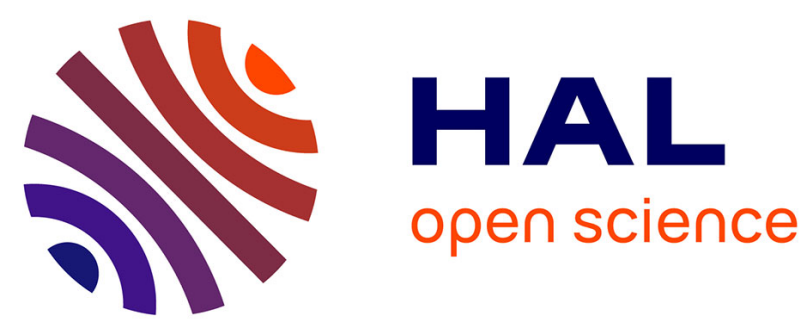

\title{
FORMULATION AND VALIDITY OF AN EQUIVALENT PHOTON APPROXIMATION IN THE TWO-PHOTON EXCHANGE MECHANISM
}

G. Bonneau, M. Gourdin, F. Martin

\section{- To cite this version:}

G. Bonneau, M. Gourdin, F. Martin. FORMULATION AND VALIDITY OF AN EQUIVALENT PHOTON APPROXIMATION IN THE TWO-PHOTON EXCHANGE MECHANISM. Journal de Physique Colloques, 1974, 35 (C2), pp.C2-109-C2-111. 10.1051/jphyscol:1974214 · jpa-00215524

HAL Id: jpa-00215524

https://hal.science/jpa-00215524

Submitted on 1 Jan 1974

HAL is a multi-disciplinary open access archive for the deposit and dissemination of scientific research documents, whether they are published or not. The documents may come from teaching and research institutions in France or abroad, or from public or private research centers.
L'archive ouverte pluridisciplinaire HAL, est destinée au dépôt et à la diffusion de documents scientifiques de niveau recherche, publiés ou non, émanant des établissements d'enseignement et de recherche français ou étrangers, des laboratoires publics ou privés. 


\title{
FORMULATION AND VALIDITY OF AN EQUIVALENT PHOTON APPROXIMATION IN THE TWO-PHOTON EXCHANGE MECHANISM
}

\author{
G. BONNEAU, M. GOURDIN and F. MARTIN \\ Laboratoire de Physique Théorique et Hautes Energies, Paris $\left({ }^{*}\right)$, France
}

\begin{abstract}
Résumé. - Nous étudions une approximation de photon équivalent dans le mécanisme d'échange de deux photons pour la diffusion Bhabha inélastique $\mathrm{e}^{+} \mathrm{e}^{-} \rightarrow \mathrm{e}^{+} \mathrm{e}^{-}+\Gamma$. Nous considérons des états finaux $\Gamma$ particuliers : $\Gamma=\mu^{+} \mu^{-} ; \Gamma=\pi^{0}, \eta, \eta^{\prime}$.
\end{abstract}

Abstract. - An equivalent photon approximation is studied for the two-photon exchange mechanism in the inelastic Bhabha scattering $\mathrm{e}^{+} \mathrm{e}^{-} \rightarrow \mathrm{e}^{+} \mathrm{e}^{-}+\Gamma$ particular cases of final state $\Gamma$ are considered : $\Gamma=\mu^{+} \mu^{-} ; \Gamma=\pi^{0}, \eta, \eta^{\prime}$.

We present here a version of the equivalent photon approximation for the two-photon mechanism in inelastic Bhabha scattering and discuss its validity.

This work was done by G. Bonneau, M. Gourdin and F. Martin (ref. [1] and [2]).

1) We have computed the diagram $(* *)$ :

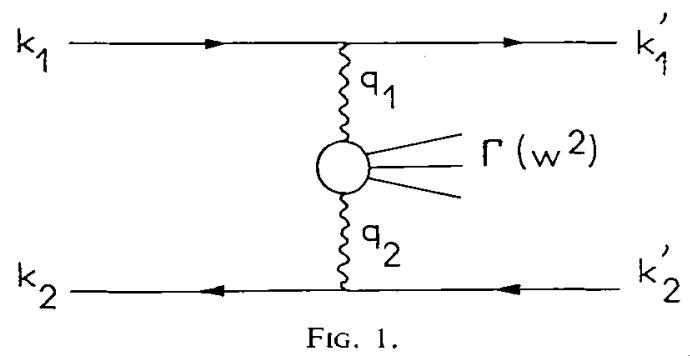

The cross section for this process involves structure functions of $\gamma^{+} \gamma \rightarrow \Gamma$. When we disregard the angular distributions of the particles of $\Gamma$ (of effective mass $W$ ) there are only 10 of them, depending on $q_{1}^{2}$, $q_{2}^{2}$ and $W^{2}$. With unpolarized electrons and positrons one can only measure 6 structure functions and one gets :

$$
\begin{aligned}
\frac{E_{1}^{\prime}}{\mathrm{d}^{3} \mathbf{k}_{1}^{\prime}} & \frac{E_{2}^{\prime}}{\mathrm{d}^{3} \mathbf{k}_{2}^{\prime}} \mathrm{d} \sigma=\frac{\alpha^{2}}{2 \pi^{4}}\left[s \left(s-\frac{1}{\left.\left.4 m^{2}\right)\right]^{1 / 2}} \frac{K W}{q_{1}^{2} q_{2}^{2}} \times\right.\right. \\
& \times\left\{\sigma_{\mathrm{TT}} K_{\mathrm{TT}}+\sigma_{\mathrm{TL}} K_{\mathrm{TL}}+\sigma_{\mathrm{LT}} K_{\mathrm{LT}}+\sigma_{\mathrm{LL}} K_{\mathrm{LL}}\right. \\
& \left.+\left(\sigma_{\|}-\sigma_{\perp}\right) K_{\mathrm{TT}}^{\mathrm{ex}}+\left(\tau_{0}+\tau_{1}\right) K_{\mathrm{LT}}^{\mathrm{ex}}\right\}
\end{aligned}
$$

(*) Postal address: Laboratoire de Physique Théorique et Hautes Energies, Université Paris VI, tour 16, 1"r étage, 4, place Jussieu, 75230 Paris Cedex 05, France.

$(* *) \Gamma$ is assumed to be free of any e $e^{-}$pair. where $m^{2}$ is the squared electron mass,

$$
s=-\left(k_{1}+k_{2}\right)^{2}
$$

and $K$ is the $\mathrm{cm}$ momentum of the $\gamma \gamma$. The indices $\lambda$ and $\lambda^{\prime}$ of the functions $K_{i \lambda^{\prime}}$, and $\sigma_{i \lambda^{\prime}}$ are the polarisation indices of the photons defined in their $\mathrm{cm}$.

The function $K_{\lambda \lambda^{\prime}}$, are known functions of $\mathbf{k}_{1}^{\prime}$ and $\mathbf{k}_{2}^{\prime}$ but obviously they depend only on 5 variables because of an overall symmetry around the incident axis of the beams. The structure functions depending only on 3 variables, the dependence of the cross section on the remaining 2 variables is known as a consequence of the two photon exchange approximation and is completely contained in the functions $K_{;} \cdot$. Then, integrating analytically over these two variables, we have computed exactly $\frac{\mathrm{d}^{3} \sigma}{\mathrm{d} q_{1}^{2} \cdot \frac{\mathrm{d}}{\mathrm{d}} q_{2}^{2} \mathrm{~d} W^{2}}$ (ref. [1]).

2) a) A quasi-real photon approximation is suggested by the poles at $q_{1}^{2}=0$ and $q_{2}^{2}=0$ and by the smallness of the electron mass which allows very small values of $q_{\mathrm{i}}^{2}$. Among the six terms of formula (1) only the first one gives a noticeable contribution to $\frac{\mathrm{d}^{3} \sigma}{\mathrm{d} q_{1}^{2} \mathrm{~d} q_{2}^{2} \mathrm{~d} W^{2}}$ for very small values of $q_{1}^{2}$ and $q_{2}^{2}$. So we consider only this term and in addition we approximate the squared amplitude $K W \sigma_{\mathrm{TT}}\left(q_{1}^{2}, q_{2}^{2}, W^{2}\right)$ by its value at $q_{1}^{2}=q_{2}^{2}=0$. These two approximations are the fundamental ingredients of the equivalent photon method (ref. [3], [4], [5]).

Then the integration over $q_{1}^{2}$ and $q_{2}^{2}$ can be done in a model independent way and we get :

$\mathrm{d} \sigma_{\mathrm{a}}=\left(\begin{array}{l}\alpha \\ \mathrm{d} W^{2}\end{array}\right)^{2} \underset{W^{4}}{2}\left(K W \sigma_{\mathrm{TT}}\right)\left(0,0, W^{2}\right) F_{\mathrm{TT}}\left(W^{2}, s\right)$. 
As we consider only large values of $s$ and $W^{2}$ as compared to $m^{2}, F_{\mathrm{TT}}\left(W^{2}, s\right)$ has the form :

$$
\begin{aligned}
& F_{\mathrm{TT}}\left(W^{2}, s\right)=\left(\log \frac{s}{m^{2}}\right)^{2} f_{1}\left(\frac{W^{2}}{s}\right)+ \\
& +\left(\log \frac{s}{m^{2}}\right) f_{2}\left(\frac{W^{2}}{s}\right)+f_{3}\left(\frac{W^{2}}{s}\right)+\mathcal{O}\left(\frac{m^{2}}{s}, \frac{m^{2}}{W^{2}}\right)
\end{aligned}
$$

Where :

(i)

$$
f_{1}(z)=\left(1+\frac{z}{2}\right)^{2} \log \frac{1}{z}-\frac{1}{2}(1-z)(3+z)
$$

is the famous function first computed by Low [6].

(ii)

$$
\begin{aligned}
f_{2}(z)= & -4\left(1-\frac{3 z^{2}}{8}\right) \log \frac{1}{z}+\frac{3}{4}(1-z)(13+z) \\
& -4\left(1+\frac{z}{2}\right)^{2}\left(\mathcal{L}_{2}(1)-\mathfrak{L}_{2}(z)\right)+\frac{z^{2}}{4}\left(\log \frac{1}{z}\right)^{2} \\
& -2(1-z)(3+z) \log (1-z)
\end{aligned}
$$

where $\mathfrak{L}_{2}$ is the Spence function :

$$
\mathfrak{f}_{2}(z)=-\int_{0}^{z} \log (1-t) \frac{\mathrm{d} t}{t} .
$$

When $s$ is large as compared to $W^{2}, F_{\mathrm{TT}}$ exhibits a logarithmic dependence in the energy:

$$
\begin{array}{r}
f_{3}\left(\frac{W^{2}}{s}\right)=-\frac{1}{3}\left(\log \frac{s}{W^{2}}\right)^{3}+\frac{3}{2}\left(\log \frac{s}{W^{2}}\right)^{2}- \\
-\frac{5}{4} \log \frac{s}{W^{2}}+c+\mathcal{O}_{3}\left(\frac{W^{2}}{s}\right)
\end{array}
$$

where

$$
c=-\frac{7}{2}+\frac{7 \pi^{2}}{6}-4 \zeta(3), \quad \zeta(3)=1.202 \ldots
$$

Let us notice that for $s$ going to infinity $F_{\mathrm{TT}}$ behaves as $\frac{2}{3}(\log s)^{3}$ (ref. [7], [5], [8]).

b) To test the validity of this approximation we have computed in a complete way the fixed $W^{2}$ and the total cross-sections [2]. The remaining contribution has been divided into two parts : one which involves the collision of a real photon and a virtual one and the other which involves the collision of two virtual photons. The two contributions to $\mathrm{d} \sigma / \mathrm{d} W^{2}$ are respectively of the type :

$$
\left(\log \frac{s}{m^{2}}\right) \mathfrak{S}_{1}\left(\frac{W^{2}}{s}, W^{2}\right)+\mathfrak{G}_{2}\left(\frac{W^{2}}{s}, W^{2}\right)
$$

and

$$
\mathfrak{S}_{3}\left(\frac{W^{2}}{s}, W^{2}\right)
$$

If the structure functions have a reasonable behaviour for large $q_{1}^{2}$ and $q_{2}^{2}$, when $s$ is large as compared to $W^{2}$, the functions $\mathcal{S}$ can be written as

$$
\begin{aligned}
\mathcal{S}_{\mathrm{j}}\left(\frac{W^{2}}{s}, W^{2}\right)=a_{\mathrm{j}}\left(W^{2}\right) \log \frac{s}{W^{2}} & + \\
& +b_{\mathrm{j}}\left(W^{2}\right)+\mathcal{O}_{\mathrm{j}}\left(\frac{W^{2}}{s}\right) .
\end{aligned}
$$

As a result we see that $f_{1}\left(W^{2} / s\right)$ and the two first terms of expansion (6) of $f_{3}\left(W^{2} / s\right)$ are model independent. Let us notice that all these model independent terms can be obtained using the following equivalent twophoton spectrum :

$$
\begin{aligned}
& N^{\gamma \gamma}\left(\omega_{1}, \omega_{2}\right)=\left(\frac{2 \alpha}{\pi}\right)^{2}\left(1-\frac{\omega_{1}}{E}+\frac{1}{2}\left(\frac{\omega_{1}}{E}\right)^{2}\right) \times \\
& \times \log \left(\frac{E W}{m \omega_{1}}\right)\left(1-\frac{\omega_{2}}{E}+\frac{1}{2}\left(\frac{\omega_{2}}{E}\right)^{2}\right) \log \left(\frac{E W}{m \omega_{2}}\right)
\end{aligned}
$$

with $\omega_{1} \omega_{2}=\frac{1}{4} W^{2}$.

The argument of the logarithms $(E W / m \omega)$ is very similar to that of original Williams' spectrum [5]. Moreover, the function $f_{2}\left(W^{2} / s\right)$ can also be obtained using the more complete spectrum :

$$
N^{\gamma \gamma}\left(\omega_{1}, \omega_{2}\right)=N^{\gamma}\left(\frac{\omega_{1}}{E}, W\right) N^{\gamma}\left(\frac{\omega_{2}}{E}, W\right)
$$

with $\omega_{1} \omega_{2}=\frac{1}{4} W^{2}$ where

$$
\begin{aligned}
N^{\gamma}\left(\frac{\omega}{E}, W\right)= & \\
=\frac{2 \alpha}{\pi}\left[\left(1-\frac{\omega}{E}\right.\right. & \left.+\frac{1}{2}\left(\frac{\omega}{E}\right)^{2}\right) \log \left[\frac{E W}{m \omega}\left(1-\frac{\omega}{E}\right)\right]+ \\
& \left.+\frac{1}{4}\left(\frac{\omega}{E}\right)^{2} \log \frac{E}{\omega}-\left(1-\frac{\omega}{E}\right)\right] .
\end{aligned}
$$

This spectrum (7) is Lorentz invariant and is the result of the use of an equivalent photon method in electroproduction when the mass of the target is small as compared to $W$.

Let us notice the difference with the Dalitz and Yennie's spectrum [11]:

$$
\begin{aligned}
N^{\gamma}(\omega)=\frac{2 \alpha}{\pi}\left[\left(1-\frac{\omega}{E}\right.\right. & \left.+\frac{1}{2}\left(\frac{\omega}{E}\right)^{2}\right) \log \left[\frac{E}{m}\left(1-\frac{\omega}{E}\right)\right]+ \\
& +\frac{1}{4}\left(\frac{\omega}{E}\right)^{2} \log \frac{2 E}{\omega}-\frac{1}{2}\left(1-\frac{\omega}{E}\right) \\
& \left.-\left(1-\frac{\omega}{2 E}\right)^{2} \log \left(1-\frac{\omega}{2 E}\right)\right]
\end{aligned}
$$


3) Some applications of these calculations have been investigated in order to test numerically this equivalent photon approximation [2].

a) The first one concerns the $\mu^{+} \mu^{-}$production where the quantum electrodynamics gives us a model for an exact and complete calculation. In this case the quasi-real photon approximation defined above works very well : the difference with the complete total cross section is at most $-2 \%$. Comparison with previous calculations of the total cross section of Brodsky, Kinoshita and Terazawa [9] and Baïer and Fadin [8] has been done [2].

b) The second application concerns the production of pseudoscalar meson $\Gamma=\pi^{0}, \eta, \eta^{\prime}$. A vector meson dominance type model has been used with one parameter $\Lambda$ in order to describe the variation of the amplitude with $q_{1}^{2}$ and $q_{2}^{2}$. It appears that the nearer the masses $\Lambda$ of the cut-off and of the produced meson are, the better our equivalent photon approximation is.

For $\pi^{0}$ production the approximation gives $3 / 4$ of the cross section for $\Lambda$ of the order of the $\rho$ meson mass (Fig. 2).

In the case of $\eta$ and $\eta^{\prime}$ production the approximation is better (the difference with the complete calculation is about $5 \%$ ).

Our calculations agree with the complete computations of Brodsky, Kinoshita and Terazawa [9].

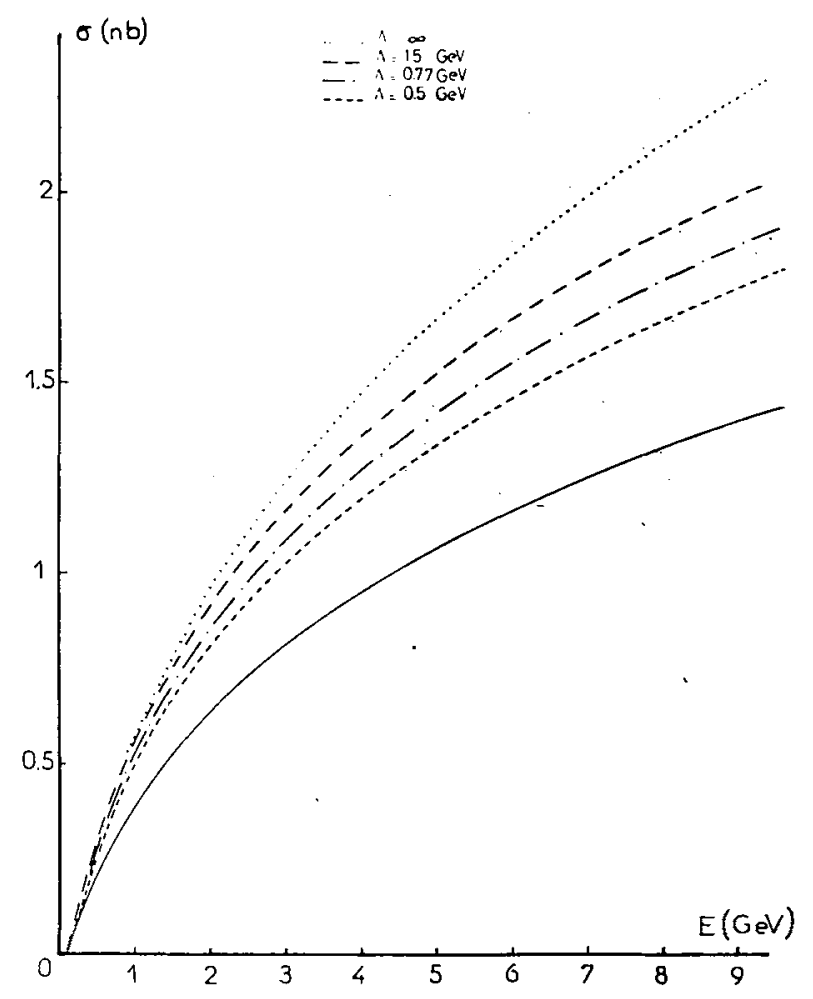

Fig. 2. - The total cross section for the process ee $\rightarrow$ ee $\pi^{0}$ as a function of $E=\frac{1}{2} \sqrt{s}$ : the curve in full line is our equivalent photon approximation $\sigma_{\mathfrak{a}}$, the other curves are the prediction of our complete calculation for different values of $A$. We use the value $\Gamma\left(\pi^{0} \rightarrow \gamma \gamma\right)=7.7 \mathrm{eV}$ (ref. [10]).

\section{References}

[1] Bonneau, G., Gourdin, M., Martin, F., Nucl. Phys. B 54 (1973) 573.

[2] Bonneau, G., Martin, F., Paris preprint PaR-LPTHE 73.4 (to be published in Nucl. Phys.).

[3] Fermi, E., Z. Phys. 29 (1924) 315.

[4] Weizsäcker, C. F. v., Z. Phys. 88 (1934) 612.

[5] Williams, E. J., Mat. Fys. Medd. Dans. Vid. Selsk. 13 $\mathrm{n}^{\mathrm{O}} 4$ (1935).

[6] Low, F. E., Phys. Rev. 120 (1960) 582.
[7] Landau, L. D. and Lifshitz, E. M., Phys, Z. Sov. 6 (1934) 244.

[8] Baíer, V. N. and Fadin, V. S., Niovo Cimento Lett. 1 (1971) 481 ; Zh. Eksp. Teor. Fiz. 61 (1971) 476 [JETP 34 (1972) 253].

[9] Brodsky, S. J., Kinoshita, T. and Terazawa, H., Phys. Rev. Lett. 25 (1970) 972 ; Phys. Rev. D 4 (1971) 1532.

[10] Particle Data Group, Rev. Mod. Phys. no 2 (1973) 45.

[11] Dalitz, R. H. and Yennie, D. R., Phys. Rev. 105 (1957) 1598. 